\title{
THE EFFECT OF LASER PULSE RISE AND FALL TIME ON MAGNETO-OPTICAL RECORDING
}

\author{
T.W. McDaniel and B.I. Finkelstein (*) \\ Optical Storage Laboratory, IBM ADSTAR, San Jose, CA 95193 \\ * Optical Product Development, IBM ADSTAR, Tucson, Arizona 85744
}

\begin{abstract}
An experimental and computer simulation study of the effect of writing laser pulse rise and fall time on magneto-optic recording performance was performed. We find that reducing the rise/fall time improves the writing efficiency and power margins, as well as improving peak shift and jitter, leading to improved detection margins
\end{abstract}

KEYWORDS: LASER, RISE TIME, FALL TIME, MAGNETO-OPTIC WRITING, JITTER, PEAK SHIFT.

\section{INTRODUCTION}

The writing process in magneto-optical (MO) recording is dependent on many parameters, including linear speed, bias field, laser pulse powertime profile, laser pedestal (baseline) power, the laser spot intensity profile, and the media thermal and magnetic characteristics. In this paper we are concentrating on the effect of the rise and fall time of the laser pulse on MO recording performance.

In order that the rise and fall time of the laser writing pulse in optical recording does not become a limiting factor in measured performance, one would like to hold those times below some fixed fraction of the data detection window $T$. For example, some existing optical recording standards require rise and fall times each to be less than $0.2 T$. As the data rate of optical storage products increases, the limitations of laser diode current driver circuits will present challenges in meeting this condition, both in test systems and in drive hardware. We performed some experimental investigations and modeling analyses to explore the threshold of performance sensitivity due to laser rise time. This paper will describe our investigation and its results.

To our knowledge, this parameter has not been studied previously, perhaps due to the experimental difficult of producing controlled variation. In our experiments, the measure of rise or fall time is the duration of the excursion between $10 \%-90 \%$ of the peak pulse power. In the modeling, since we used trapezoidal pulse shapes, the base-peak transition time is the rise or fall time.

\section{EXPERIMENTAL}

Tests were done on a precision test stand using $780 \mathrm{~nm}, 0.55 \mathrm{NA}$ optics. Writing of pulse position modulation test patterns was done at 2400 RPM on a 5.25 inch diameter MO disk using 20 ns writing pulses. Rise time was varied from 5 to 9 ns by modifying the capacitance in laser driver circuitry. Recording performance as a function of write power, as indicated by signal amplitude, time domain jitter, and worst-case peak shift, was measured for each rise time. Rise times less than 5.4 ns could not be evaluated because of tester hardware limitations, and rise times much longer than 9 ns are unreasonable for a $20 \mathrm{~ns}$ pulse. At each rise time, the pulse duration was adjusted to maintain a 20 ns FWHM.
Due to some "ringing" near the peak of the pulse power, the energy content of experimental pulses was monitored using its time integral.

\section{MODELING}

Computer simulations of the MO recording experiments were carried out with a program which develops an axisymmetric 3D finite element thermal impulse solution for the test disk, combined with a superposition program to develop the full thermal field during arbitrary writing sequences [1]. Mark domain wall location was assumed to coincide with the 175 degree $C$ isotherm of temperature elevation above ambient for the cumulative maximum temperature at any point in the MO film. The thermal field solution was typically done on a rectangular $2 \mathrm{D}$ mesh with $\Delta x=\Delta y=50 \mathrm{~nm}$, with a time-step of either 1 or 3 ns. In preparation for readback signal simulation, a mesh refinement in each linear dimension by a factor $f$ (normally 5 or 10 ) was performed by a two-dimensional cubic spline interpolation of the maximum temperature surface $T(x, y)$. Such an interpolation was confirmed to be completely satisfactory due to the smooth character of the cumulative maximum thermal field. The length and width of the mark-defining isotherm could be accurately calipered from the interpolated mesh. Readback was simulated with a simple spot-mark overlap model, with an $\mathrm{f}^{2}$ factor improvement in signal resolution provided by the thermai interpolation.

Conditions identical to those used experimentally at the outside diameter were simulated. Separate runs were made to estimate the signal amplitude of high and low frequency tone patterns. An optimum write power for each pulse rise/fall time could be accurately interpolated by relating the physical mark length associated with the peak of the high frequency tone pattern. At that optimum write power, a test pattern used experimentally to test worst-case peak shift and mark-to-mark jitter was modeled in separate simulations. Peak shift determinations required the greatest precision in the simulation, since the spatial and temporal shifts sought are not much larger than the minimum model resolution. available for tolerable computing throughput.

A peak shift estimate is done in two steps. In a first writing pass, an isolated mark is written to establish a reference for the estimation of the shift of the signal peak from the second mark of the peak 
Table 1. Risetime data - modeling \& experiment $(v=14.6 \mathrm{~m} / \mathrm{s})$

\begin{tabular}{lllllll} 
& & Modeling & & \multicolumn{3}{c}{ Experiment } \\
t(rise/fall,ns) & Pw,opt & PS(nm) & PS(ns) & t(rise/fall) & Pw,opt & PS(ns) \\
$8.0 / 9.5$ & 10.82 & 25.7 & 1.76 & $8.0 / 9.5$ & 9.7 & 1.60 \\
$6.4 / 8.3$ & 10.70 & 24.7 & 1.69 & $6.4 / 8.3$ & 8.75 & 1.68 \\
$5.5 / 6.8$ & 10.59 & 23.4 & 1.60 & $5.5 / 6.8$ & 8.8 & 1.55 \\
$5.2 / 5.4$ & 10.54 & 23.5 & 1.61 & $5.2 / 5.4$ & 8.25 & 2.20
\end{tabular}

shift pattern dimark. In a second writing pass, the dimark of the peak shift pattern is written and the location of the readback pulses noted (see Fig. 5). An absolute shift of the position of the second dimark signal peak can be calculated from the results of the two writing/readback passes. The peak shift simulated is the composite effect of simple readback intersymbol interference and thermal interaction between the marks of the dimark. Of course, these separate contributions could be identified in the computer simulation, if desired. Jitter was not simulated in this paper [2].

\section{RESULTS}

\section{Experiment}

We collected experimental data for the high frequency (HF) tone pattern signal amplitude versus write power with write pulse rise/fall time as a parameter. In general, the power at which the peak occurs ("optimum write power") is shifted toward lower power for rapid rise/fall times. The low frequency signal amplitude at the optimum power is highest for the rapid rise/fall time case (see Table 1). The HF signal jitter for the fastest rise/fall time is clearly the lowest of the four cases, as Figure 1 shows. However, for the three slower rise/fall times, the minimum jitter is essentially the same, and only $0.1 \mathrm{~ns}$ higher than for the fast rise time. We also measured the peak shift of the second peak of the dimark in the peak shift pattern as a function of write power for the four rise/fall time cases, as shown in Table 1. Figure 2 shows a recording performance figure of merit (FOM) versus write power with pulse rise/fall time as a parameter. We define

$$
\mathrm{FOM}=\left[\left(\mathrm{T}_{W} / 2\right)-\mathrm{PS}\right] \div(\sigma \div \sqrt{2}),
$$

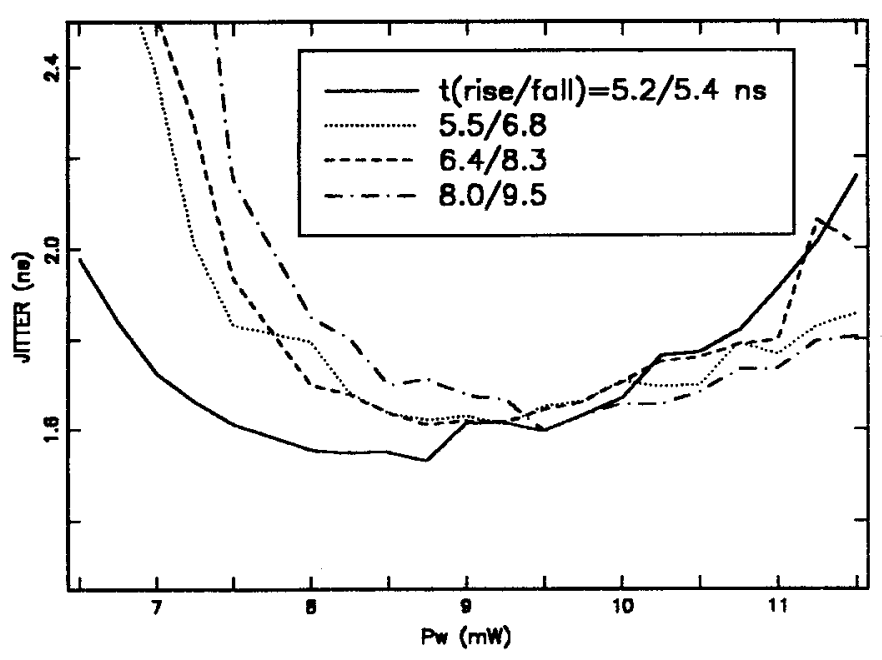

Figure 1. Measured mark-to-mark jitter vs, write power. where $\mathrm{T}_{\mathrm{w}}=$ clock window, $\mathrm{PS}=$ peak shift, and $\sigma=$ the standard deviation of the mark-to-mark timing distribution, i.e. jitter. Little difference in the: peak value of FOM is seen for the four rise times, although the aforementioned power dependence of the optimum value shows up. The difference in jitter values with rise time may be somewhat offset by compensating differences in peak shifts at the respective power values of maximum FOM. In any case, the range of write powers over which a given FOM is reached (a measure of power margin) definitely increases with decreasing rise time.

\section{Modeling}

Figure 3 shows the modeled dependence of HF tone signal amplitude and physical mark length on write power. From a series of such plots versus rise/fall time, one learns that the signal peak occurs at an edge-to-edge mark length equal to a fixed fraction of the along-track mark pitch. The optimum powers derived from such data are summarized in Table 1. The writing pulses used were trapezoidal, with a baseline power of $0.8 \mathrm{~mW}$ and a pulse FWHM of 20 ns maintained for all experiments. Symmetric rise and fall times were used. Figure 4 shows a family of mark length versus write power curves with pulse rise/fall time as a parameter. Since written marks of a certain length correspond the peak in the tone signal amplitude versus write power curve, the trend with rise/fall time illustrated in this figure explains the observation that optimum power increases monotonically with rise/fall time. It appears that power for writing is most efficiently delivered to the disk with pulses of short rise and fall time.

Figures $5 \mathrm{a}$ and $5 \mathrm{~b}$ show an example of a modeled isolated dimark and the readback signisl from that

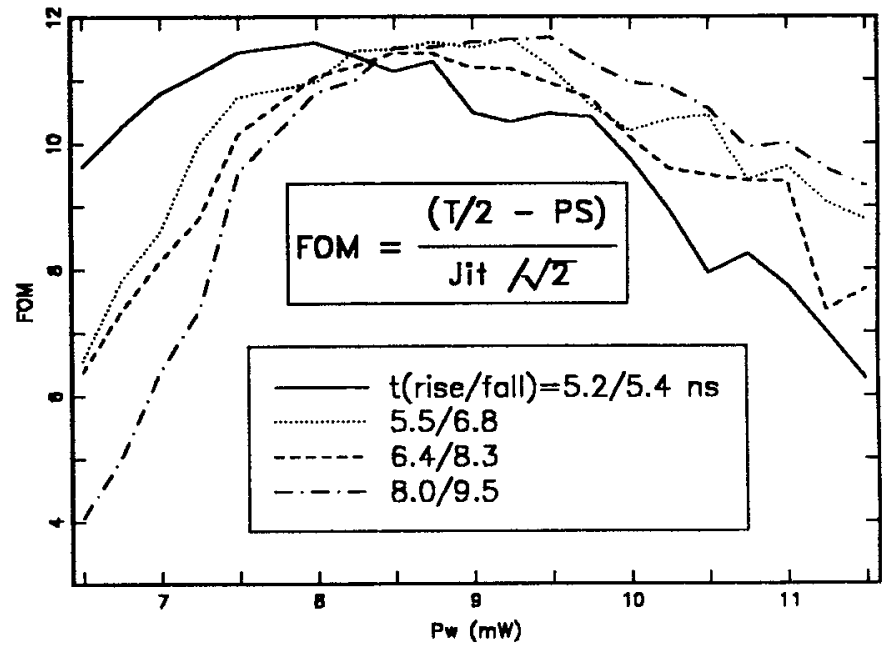

Figure 2. Measured recording performance figure of merit vs write power. 


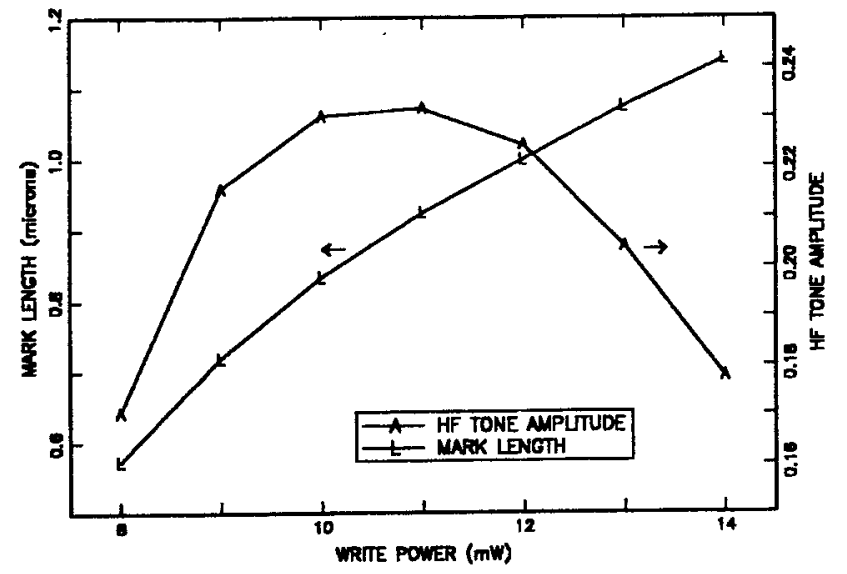

Figure 3. Modeled dependence of high frequency tone signal amplitude and physical mark length on write power.

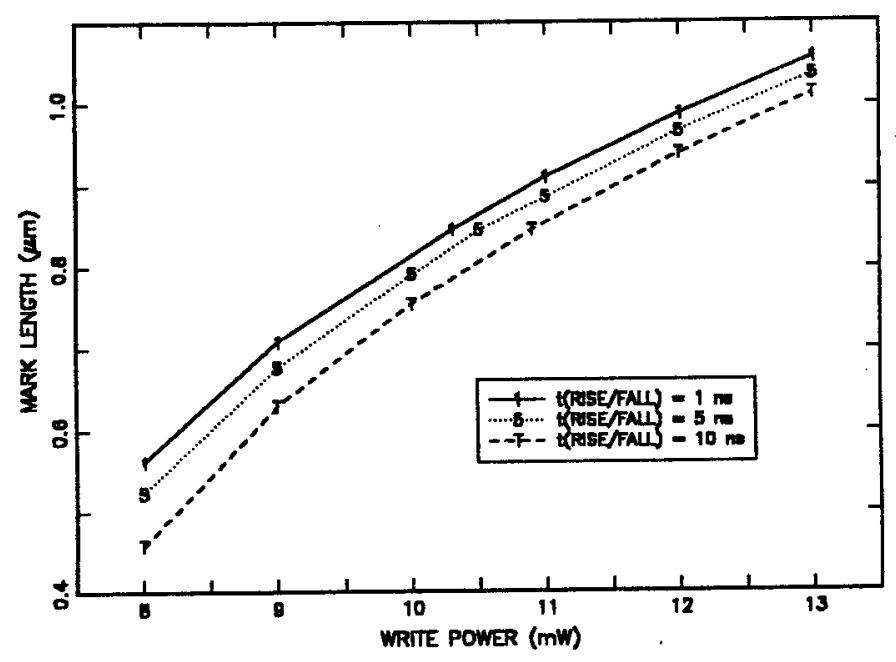

Figure 4. A family of mark length versus write power curves.

feature. One clearly sees the effect of thermal interaction between the closely spaced marks, as the second mark is written on a track region preheated by the first mark writing event, provided that the writing spot displacement time exceeds the speed of the thermal spreading in the disk. The preheating causes the second mark to be enlarged, and slightly displaced toward a lower $x$-value than if it had been written as an isolated feature. The total perceived peak shift in the peak location of the readback signal is further increased by the inability of the finite sized reading spot to perfectly resolve the closely spaced mark pair. This intersymbol interference pushes the successive peaks of the unipolar MO signal closer together - opposite the effect in magnetic recording where a bipolar signal response results from closely spaced magnetic transitions.

The general behavior of dimark peak shift with pulse power and rise time is shown in Figures $6 a$ and $6 \mathrm{~b}$. While there is a clear monotonic increase with pulse power, it is not apparent how neak shift at the optimum write power (operating power) depends on rise/fall time. Figure 7 shows this relationship for both the experiment and modeling. While the modeling clearly exhibits a monotonic relationship between dimark peak shift and write pulse rise/fall
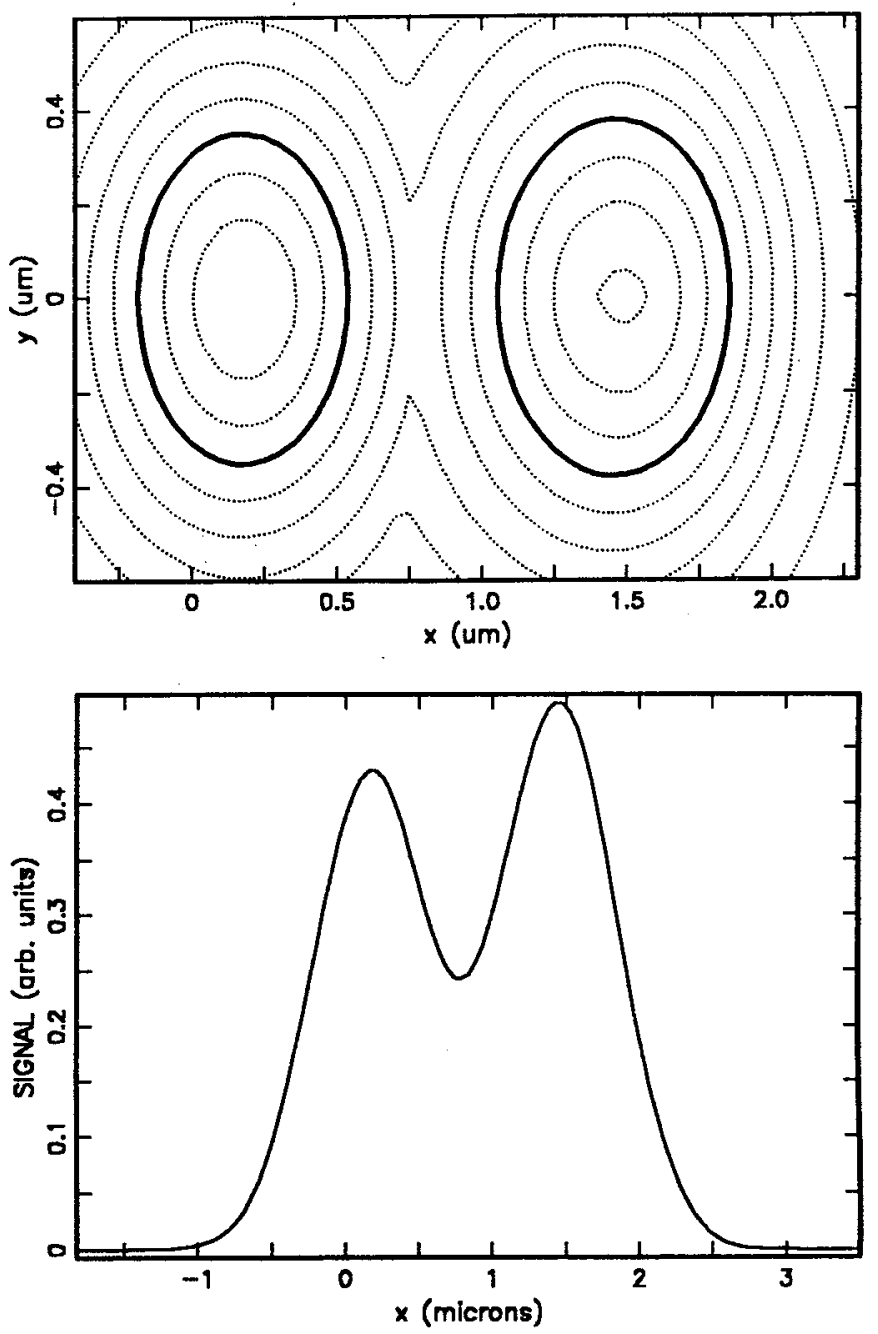

Figure 5. (a) Isotherms in MO film plane. Heavy boundary shows written dimark at 175 degC above ambient. (b) Readout signal from dimark.

time at optimum power, the experimental result is not so clear. The monotonic relation is intuitively expected on physical grounds, so the experimentally observed minimum at intermediate rise time is unexpected. To examine the possibility that the experimental result was due to the asymmetric rise and fall times, we modified the model to include this feature. Results for this case are also shown in Fig. 7. Again, the last three of the four experimental points could be reasonably reproduced with this version of the model, but the asymmetric rise and fall time is apparently not an important factor.

\section{CONCLUSIONS}

We have found a relatively weak but clear dependence of MO recording performance on write pulse rise/fall time over the range 1-10 ns. The primary effect of varying rise time is a change in optimum write power and write power margin. As rise time decreases, required pulse power decreases as does the total energy required to write a mark. Also, decreasing rise time extends the range of usable write powers, in both relative and absolute terms. This is evidently due to closer adherence to the adiabatic limit for power delivery to the disk for short rise/fall time, in which heat loss through con- 
duction during the writing event can be neglected. As the writing pulse becomes more distributed in time with slower rise/fall time, more heat is lost through conduction during the writing.

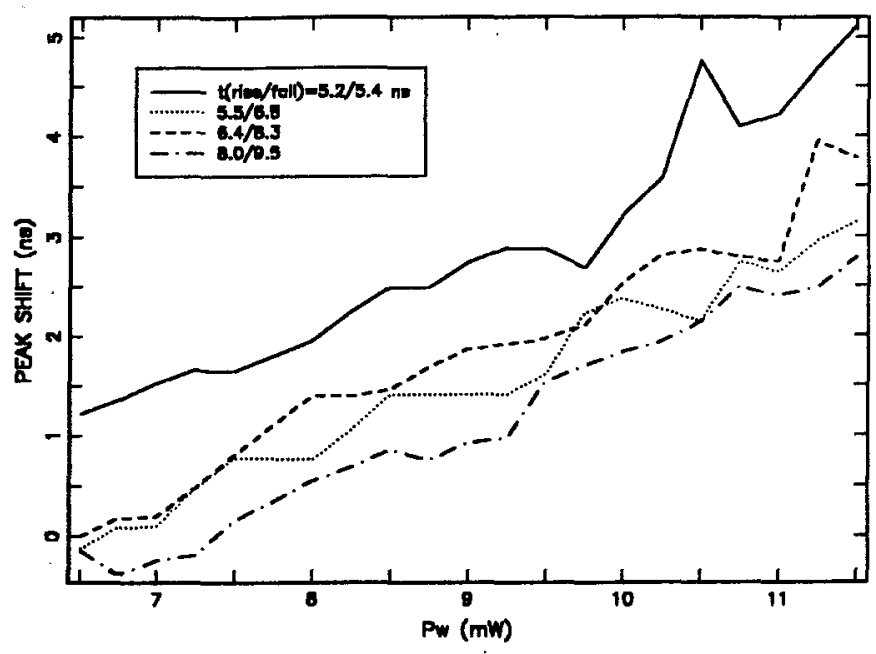

Figure 6a. Experimental dimark peak shift vs. power and rise time.

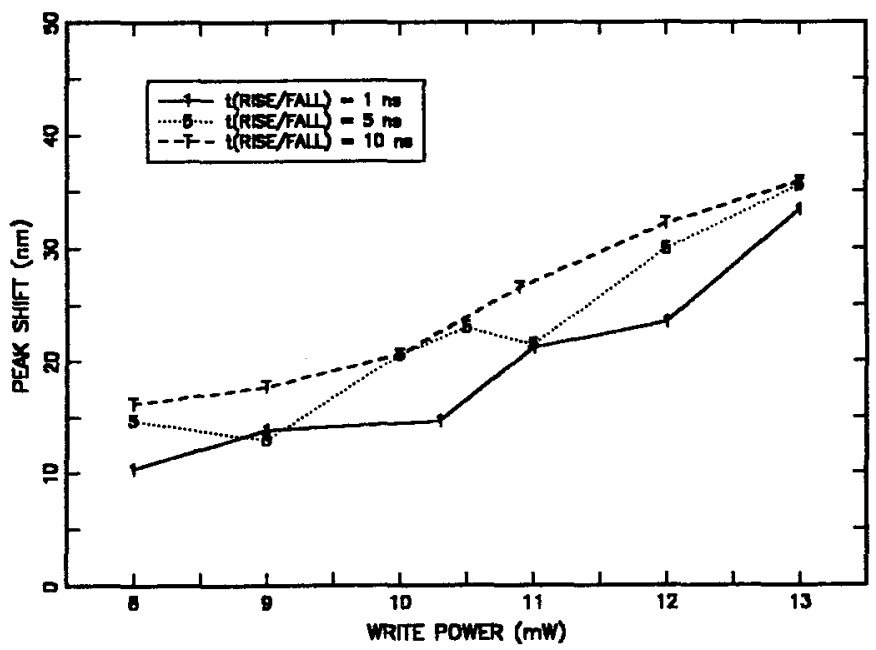

Figure $6 \mathrm{~b}$. Modeled dimark peak shift vs. power and rise time.

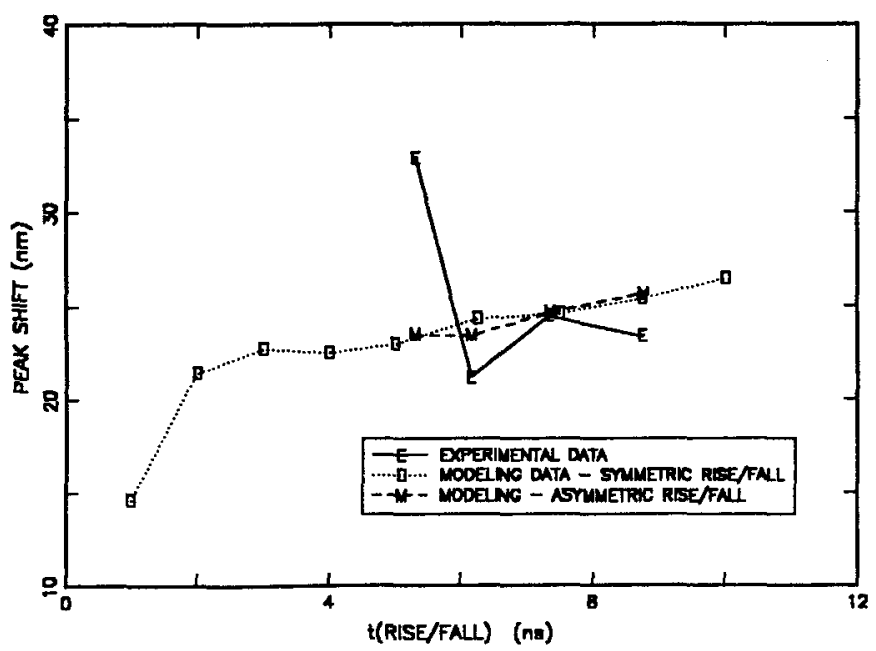

Figure 7. Dependence of peak shift at optimum write power on pulse rise and fall time; one experimental and two modeling cases.
The modeling work is supportive of the experimental findings, at least qualitatively, and it also provides explanatory power. The simulation of write pulse rise/fall time effects challenges a numeric recording model because of the extreme precision required. Experimentally measured values of peak shift at $14.6 \mathrm{~m} / \mathrm{s}$ lay in the range 1.4 to $2.2 \mathrm{~ns}$, corresponding to spatial distances of 20 to $32 \mathrm{~nm}$. This shows why the spatial discretization of the numerical model had to be reduced down to the $5 \mathrm{~nm}$ range. Also, to accurately model rise/fall times down to $1 \mathrm{~ns}$ in $1 \mathrm{~ns}$ increments required special attention to accurate representation of pulse power sampling and delivery to the disk over the pulse duration.

The importance of rise time is expected to be greater for pulse width modulation recording since slower rise/fall times cause more uncertainty in mark edge location.

\section{REFERENCES}

[1]T.W. McDaniel and F.O. Sequeda, "Design and Material Selection for a Thin Film Magneto-Optic Disk", Appl. Phys. Commun. 11, 427 (1992).

[2]T.W. McDaniel, "Simulation of Bit Jitter in Magneto-Optical Recording", J. Appl. Phys. 63, 3859 (1988). 